\title{
Intragenic and large NIPBL rearrangements revealed by MLPA in Cornelia de Lange patients
}

\author{
Silvia Russo ${ }^{\star, 1}$, Maura Masciadri ${ }^{1}$, Cristina Gervasini ${ }^{2}$, Jacopo Azzollini ${ }^{2}$, Anna Cereda ${ }^{3}$, Giuseppe Zampino ${ }^{4}$, \\ Oskar Haas $^{5}$, Gioacchino Scarano ${ }^{6}$, Maja Di Rocco ${ }^{7}$, Palma Finelli ${ }^{1}$, Romano Tenconi ${ }^{8}$, Angelo Selicorni ${ }^{3}$ \\ and Lidia Larizza ${ }^{1,2}$
}

Cornelia de Lange syndrome (CdLS) is a rare multisystemic congenital anomaly disorder that is characterised by intellectual disability and growth retardation, congenital heart defects, intestinal anomalies, facial dysmorphism (including synophyris and high arched eyebrows) and limb reduction defects. Mutations in three cohesin-associated genes encoding a key regulator (NIPBL, chr 5p13.2) and one structural component of the cohesin ring (SMC1A, chr Xp11) occur in about 65\% of CdLS patients. NIPBL is the major causative gene, and accounts for $40-60 \%$ of CdLS patients as shown by a number of mutational screening studies that indicate a wide mutational repertoire of mainly small deletions and point mutations. Only a few data are available concerning the occurrence of large NIPBL rearrangements or intragenic deletions or duplications involving whole exons. We used multiplex ligation-dependent probe amplification (MLPA) to study 132 CdLS patients negative to the standard mutation NIPBL test out of a cohort of 200 CdLS patients. A total of 7 out of 132 patients were found to carry NIPBL alterations, including two large gene deletions extending beyond the gene, four intragenic multi- or single-exon deletions and one singleexon duplication. These findings show that MLPA leads to a 5.3\% increase in the detection of mutations when used in addition to the standard NIPBL scan, and contributes per se to the molecular diagnosis of 3.5\% (7/200) of clinically diagnosed CdLS patients. It is recommended that MLPA be included in the CdLS diagnostic flow chart.

European Journal of Human Genetics (2012) 20, 734-741; doi:10.1038/ejhg.2012.7; published online 22 February 2012

Keywords: Cornelia de Lange; NIPBL; MLPA; intragenic deletion/duplication; large rearrangements; transcript analysis

\section{INTRODUCTION}

Cornelia de Lange syndrome (CdLS; MIM\#122470, 300590) is a multi-system malformation/intellectual disability disorder caused by the developmental and post-natal defects of the cohesin pathway. ${ }^{1-3}$ Alterations in the nipped-B-like NIPBL (MIM\#608667) and structural maintenance of chromosomes 1A SMC1A (\#300040) genes, which encode a cohesin regulator and a structural subunit of the cohesin complex, respectively, account for 40-60 and 5\% of CdLS patients, ${ }^{4-7}$ but other causative genes are thought to be involved in CdLS patients who are negative for mutations in the known genes. Consequently, especially in the case of the major NIPBL gene, the diagnostic test should be refined by means of a multi-method approach and sequencing extended to the non-coding regions in order to reveal the entire fraction of patients with gene mutation and identify definitive negative patients suitable for next-generation sequencing aimed at detecting novel genetic determinants. Genomic and genetargeted techniques such as array-comparative genomic hybridisation (a-CGH) and multiplex ligation-dependent probe amplification (MLPA) can reveal some NIPBL imbalances overlooked by the standard test, although both techniques have only been sporadically used to screen CdLS patients. In patients with a CdLS-like clinical diagnosis who are negative to NIPBL scan, a-CGH has mainly revealed de novo imbalances in various genomic regions that maybe too large to identify new CdLS genes. ${ }^{8-10}$ Given the length of the NIPBL gene and the plausible failure of its splicing mechanism, MLPA undoubtedly seems to be the most promising additional means of optimising the diagnostic mutation rate. However, only three MLPA-detected CdLS patients have so far been described in the literature, one of which was fortuitously found in the context of a study aimed at evaluating the increased DNA damage sensitivity of CdLS syndrome cell lines. ${ }^{11}$ The other two patients, one with an intragenic deletion encompassing NIPBL exons $41-42$ and the other with a large deletion affecting the last 13 exons of NIPBL and extending beyond it, were identified from cohorts of 50 and 11 CdLS patients, respectively, who were negative to the standard gene test. ${ }^{12,13}$ In order to evaluate the fraction of CdLS patients undiagnosed by NIPBL denaturing high-performance liquid chromatography (DHPLC)/sequencing, we used MLPA to process systematically 132 out of 200 CdLS patients negative to the test. Seven of the negative patients were found to bear a previously undetected NIPBL alteration. We also characterised at cDNA level three intragenic $N I P B L$ rearrangements and refined the genomic mapping of two large deletions, respectively, extending to the $5^{\prime} \mathrm{UTR}$ and $3^{\prime} \mathrm{UTR}$ of the NIPBL gene.

\footnotetext{
${ }^{1}$ Istituto Auxologico Italiano, Laboratory of Medical Cytogenetics and Molecular Genetics, Milan, Italy; ${ }^{2}$ University of Milano, Department of Medicine, Surgery and Dentistry, Medical Faculty, Milano, Italy; ${ }^{3}$ Paediatrics Clinical Genetics, MBBM AO S Gerardo Monza Foundation, University of Milano Bicocca, Monza, Italy; ${ }^{4}$ European University, Department of Paediatrics, Roma, Italy; ${ }^{5}$ Department of Paediatric Haematology and Oncology, St Anna Children's Hospital, Wien, Austria; ${ }^{6} \mathrm{G}$ Rummo Hospital, Medical Genetics Department, Benevento, Italy; ${ }^{7}$ Unit of Rare Diseases, Department of Paediatrics, Gaslini Institute, Genoa, Italy; ${ }^{8}$ University of Padova, Genetics Paediatrics, Padua, Italy *Correspondence: Dr S Russo, Istituto Auxologico Italiano, Laboratory of Medical Cytogenetic and Molecular Genetics, Via Zucchi 18, Cusano Milanino, Milan 20095, Italy. Tel: +39 2 619113036; Fax +39 2 619113033; E-mail: s.russo@auxologico.it

Received 13 April 2011; revised 5 December 2011; accepted 23 December 2011; published online 22 February 2012
} 


\section{METHODS}

Subjects

A cohort of 200 patients with a clinical diagnosis of CdLS (108 males and 92 females, ranging from newborn to adult age) has so far been referred to our Institute for the genetic screening of the NIPBL and SMC1A genes. The CdLS patients of our cohort had been evaluated by different clinical geneticists, all experienced in CdLS, who used the parameters of Kline $e^{~} a^{14}$ and, whenever possible, were given an overall clinical score. Peripheral blood samples were obtained from all the patients following their or their parents' informed consent.

\section{DHPLC and direct sequencing}

The genomic DNAs underwent the mutational screening of the NIPBL gene (RefSeq NM_133433) by means of DHPLC using intronic primers flanking the whole coding sequence and the predicted promoter region (http:// www.cbs.dtu.dk/services/Promoter/, promoter 2.0 prediction) set up for polymerase chain reaction (PCR) and DHPLC temperature conditions. Heteroduplex fragments underwent direct sequencing. The detailed protocol is available on request.

\section{MLPA analysis}

The SALSA P141/P142 NIPBL MLPA kit (MRC-Holland, Amsterdam, The Netherlands) was used in accordance with the manufacturer's instructions. The parents' DNA was analysed to establish the de novo origin of the rearrangements. cDNA was analysed when sufficient material was available. Genomic real-time qPCR was carried out to validate the MLPA-detected duplication.

\section{SNP analysis}

The segregation of the centromeric-to-telomeric SNPs rs12658985, rs11740860, rs10035888, rs10491374, rs1004202, rs10042632 and rs10036454 from the parents to the probands was checked by sequencing in order to define the largest NIPBL deletion.

\section{cDNA analysis of mutant alleles}

Total RNA was extracted from fresh peripheral blood or lymphoblastoid cell lines using the Trizol procedure (Life Technologies, Grand Island, NY, USA). cDNA for RT-PCR was obtained using the SuperScript First-Strand Synthesis System (Invitrogen Ltd, Inchinnan Business Park, UK), with the primers being selected by means of Primer Express software. Primers amplifying a product from untranslated exon 1 to exon 3 (fw: $5^{\prime}$-ATTATAGTCTCTCGCCAC- $3^{\prime}$ and rev: 5'-GTAGTTGTAGCAGGTAAAG-3'), from exon 24 to exon 28 (fw: $5^{\prime}$-AC TGTTGCTGCACGGCTAA- ${ }^{\prime}$ and rev: $5^{\prime}$-CTCGTTGCATATCAAGCCTTG- ${ }^{\prime}$ ) and from exon 2 to exon 4 (fw: $5^{\prime}$-ACTGTTGCTGCACGGCTAA-3' and rev: $5^{\prime}$-CTCGTTGCATATCAAGCCTTG- $3^{\prime}$ ) were used to characterise deletions. The PCR products from the cDNAs were loaded on $3 \%$ agarose gel, eluted by means of GFX (Gel Heathcare, Buckinghamshire, UK) and sequenced using the above primers in order to evaluate the presence and ratio of the aberrant transcripts in comparison with the wild type.

\section{Real-time qPCR assay}

For the real-time qPCR assay of genomic DNA, the SYBR Green protocol (SYBR GREEN, PCR Master Mix, Applied Biosystem, Warrington, UK) was applied using primers located within exon 32 (fw: $5^{\prime}$-CTTTGATTCTTTTCA TCACCCTTA- $3^{\prime}$ and rev: $5^{\prime}$-GCAGTTGCTCAAACCAGTCA- $3^{\prime}$ ). Amplicons were run as triplicate in separate tubes to allow the quantification of the NIPBL gene, normalised to the endogenous PGK1 (phosphoglycerate kinase 1) gene and amplified using primers fw: $5^{\prime}$-GAAGTGGAGAAAGCCTGTGCC- $3^{\prime}$ and rev: $5^{\prime}$-TTCTTCCTCCACATGAAAGCG-3'. The gene copy number of the investigated sample was then estimated using a calibrator sample of normal, healthy control DNA. The results were quantified using the comparative threshold cycle method.

\section{Clone preparation}

All of the BAC clones were purchased from Invitrogen S.r. l. DNA was obtained from a single colony grown in $10 \mathrm{ml}$ of LB medium, supplemented with $12.5 \mu \mathrm{g} / \mathrm{ml}$ chloramphenicol (High Performance CCD Camera (COHU,
Poway, CA, USA) and visualised using McProbe software (Applied Imaging, PowerGene, League City, TX, USA)) in accordance with standard procedures.

\section{Cell culture and chromosome preparations}

Phytohemagglutinin-stimulated peripheral blood lymphocytes from the samples were set up in culture using Chromosome Kit 'Synchro' (Celbio, Milan, Italy) and modified RPMI (Irvine Scientific, Santa Ana, CA, USA) plus $5 \%$ foetal calf serum (Gibco, Grand Island, NY, USA). The cultures were stopped with colchicine after $72 \mathrm{~h}$. The chromosome preparations were obtained using standard methods.

\section{FISH}

The BAC probes were labelled with digoxigenin-dUTP (Roche Diagnostic, Mannheim, Germany) using a nick translation kit (Roche Diagnostic). The FISH experiments were performed using standard procedures. ${ }^{15}$ The chromosomes were counterstained with DAPI in antifade (Vectashield Mounting medium, Vector Laboratories Inc., Burlingame, CA, USA), and then visualised using a Leitz DM-RB microscope equipped for DAPI and FITC/TRITC epifluorescence optics. The images were captured by means of a CCD camera (Hamamatsu 3CCD Camera, C5810, Hamamatsu City, Japan), and visualised using McProbe software.

\section{a-CGH analysis}

Genomic DNA was extracted from probands' and parents' whole blood using the GenElute Blood Genomic DNA kit (Sigma-Aldrich, St Louis, MO, USA) in accordance with the manufacturer's instructions. The reference was pooled DNA from the peripheral blood of 10 healthy donors (Promega, Madison, WI, USA) who were gender-matched to the samples. The genome scan was performed using the Human Genome CGH Microarray Kit 244K (Agilent Technologies, Palo Alto, CA, USA), which consists of 236000 60-mer oligonucleotide probes covering the entire genome at an average spatial resolution of $\sim 30 \mathrm{~kb}$. The samples were labelled and hybridised following the protocols provided by Agilent. The arrays were analysed using the Agilent Scanner Control (v 7.0) and Feature Extraction software (v 9.5.1). Graphical overviews were obtained using CGH Analytic software (v 4.0.81). A log ratio plot between the test and reference genomic DNA was assigned so that aberrations in test DNA copy numbers at a particular locus were observed as a deviation of the ratio plots from a modal value of 0 . Aberration calls were identified using the ADM-2 algorithm. An in silico analysis of the observed unbalanced region was made using the March 2006 release of the UCSC Genome Browser (http://genome.ucsc.edu/) and the Database of Genomic Variants (http://projects.tcag.ca/variation).

\section{RESULTS}

A cohort of 200 patients ranging from earliest infancy to adult age with a clinical diagnosis of CdLS underwent molecular screening of the NIPBL gene. They were divided into three subgroups on the basis of their clinical score (severe $>22$, moderate $15-22$, and mild $<15$ ) as previously described in the case of 62 of them. ${ }^{7}$ NIPBL DHPLC and sequencing of the coding and flanking regions plus a portion of the $5^{\prime}$ UTR allowed us to identify 68 patients with gene mutations, including those previously described: ${ }^{7} 29$ truncating (19 frameshift and 10 nonsense), 13 missense and 23 splicing mutations, and 2 in-frame deletions affecting the coding and consensus sequence, and 1 mutation in the $5^{\prime} \mathrm{UTR}$, for an NIPBL mutation rate of $34 \%$. This low rate may be explained by the clinical heterogeneity of this cohort that includes a considerable number of patients with a very mild phenotype. All of the samples that were negative to the standard NIPBL scan were processed using the MLPA test in order to evaluate the occurrence of whole exon deletions/duplications. This revealed six deletions: one involving the first 10 exons of NIPBL and $2 \mathrm{Mb}$ upstream (patient 1), one whole gene deletion extending $0.5 \mathrm{Mb}$ downstream (patient 7), the intragenic deletion of three exons (25-27) in patient 4, and three 


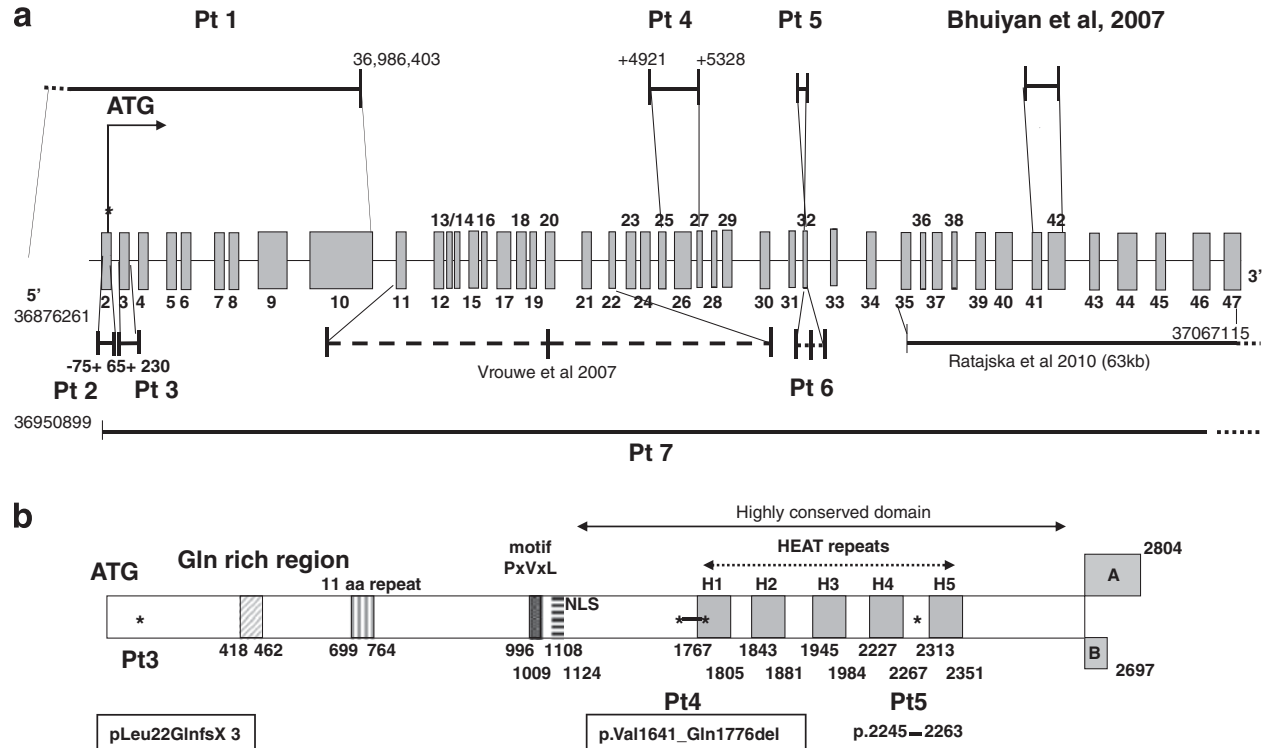

Figure 1 Overall picture of MLPA-detected large deletions/duplications involving the NIPBL gene. (a) Schematic illustration of the NIPBL gene: the black bars mark the genomic deletions in patients 1 and 7 and the skipped exons in patients 2, 3, 4, and 5; the findings relating to two previously described patients in the literature are also included. The dashed bars indicate the duplications of patient 6 and the patient reported by Vrouwe; the large genomic duplications reported by Yan et al ${ }^{16}$ are not included because they are out of scale. The genomic boundaries of the rearrangements of pts 1 and 7 and cDNA boundaries of pts 2, 3, and 4 are indicated at the ends of each bar. (b) Schematic illustration of the NIPBL protein with its putative domains and motifs: the asterisks indicate the localisation of the predicted deleted amino-acid residues (patients 4 and 5) and the protein truncation start site (patient 3 ).

\section{Table 1 Clinical features of CdLS patients in this study}

\begin{tabular}{|c|c|c|c|c|c|c|c|c|c|c|c|c|c|}
\hline & & & & & & & & & & & & Mutc & ation \\
\hline$P t$ & Sex & year & dysmorphism & IUGR & $P N G R$ & reduction & malformations ${ }^{a}$ & disability $^{b}$ & Speech & Medical complications & Clinical score ${ }^{c}$ & Localisation & Type \\
\hline 1 & M & 36 & + & + & + & + & - & Severe & Absent & GER, deafness & 38 Severe & 5'UTR ex 1-10 & $\begin{array}{l}\text { Large } \\
\text { deletion (2 Mb) }\end{array}$ \\
\hline 2 & $\mathrm{~F}$ & 16 & + & - & - & - & - & Moderate & Retarded & GER, obesity & 20 Mild & Ex 2 & Deletion \\
\hline 3 & $\mathrm{~F}$ & 3 & + & - & + & - & - & Moderate & Absent & GER & 18 Mild & Ex 3 & Deletion \\
\hline 4 & M & 4 & + & + & + & + & - & Severe & Absent & GER & 23 Severe & Ex 25-27 & Deletion \\
\hline 5 & M & 12 & + & + & + & - & $2(\mathrm{G} ; \mathrm{U})$ & Severe & Absent & Myopia deafness & 34 Severe & Ex 32 & Deletion \\
\hline 6 & M & - & + & - & NA & NA & NA & NA & NA & NA & NA & Ex 32 & Duplication \\
\hline 7 & $\mathrm{~F}$ & 6 & + & + & + & + & $1(\mathrm{~B})$ & Moderate & Retarded & $\begin{array}{l}\text { GER, and myopia, } \\
\text { full body hirsutism }\end{array}$ & 26 Severe & Ex 2-47 3'UTR & $\begin{array}{l}\text { Large } \\
\text { deletion }(515 \mathrm{~kb})\end{array}$ \\
\hline $8^{d}$ & $\mathrm{~F}$ & 1,8 & + & - & + & + & $2(\mathrm{C} ; \mathrm{U})$ & Severe & NA & $\begin{array}{l}\text { GER, full body hirsutism, } \\
\text { hypertonic, seizures, apnea }\end{array}$ & 28 Severe & Ex 41-42 & Deletion \\
\hline $9^{e}$ & $\mathrm{~F}$ & 14 & + & - & + & + & $3(\mathrm{C} ; \mathrm{K} ; \mathrm{U})$ & Severe & Absent & GER & 30 Severe & Ex 35-47 3'UTR & $\begin{array}{l}\text { Large } \\
\text { deletion (63 kb) }\end{array}$ \\
\hline
\end{tabular}

Abbreviations: B, brain; G, genitalia; GER, gastroesophageal reflux; GI, gastrointestinal; IUGR, intrauterin growth retardation; NA, not available; PNGR, postnatal growth retardation; U, urinary. aNumbers refer to different apparatus/anatomic sites.

bMild $<15$; moderate $15-22 ;$ severe $>22$

CAccording to Kline et al. ${ }^{14}$

dBhuiyan et al. ${ }^{12}$

eRatajska et al. ${ }^{13}$

single exon deletions in patients 2 (exon 2), 3 (exon 3 ) and 5 (exon 32), and a duplication of exon 32 in patient 6 . The parents of all of the patients with deletions were non-carriers; the deletions were considered de novo. The identified NIPBL rearrangements are shown in Figure 1a, which also includes two previous MLPA-detected intragenic deletions ${ }^{12,13}$ and one duplication. ${ }^{11}$ Five large genomic duplications on chromosome 5 involving the NIPBL gene have also been reported in patients that share some of the features of CdLS but not its facial gestalt. ${ }^{16}$ As MLPA does not allow the precise mapping of the rearrangements or predict the coding of a deleted truncating or in-frame protein, we investigated the mutations at transcript level in three patients for whom biological samples were available (see next section). Figure $1 \mathrm{~b}$ shows the putative position of the deletions on the protein and the various delangin domains affected. In the case of NIPBL whole gene deletion (patient 7), deletion of the first 10 exons (patient 1) or the deletion of exon 2 (patient 2), the physiological start codon is skipped, thus predicting that the protein is not translated. We consulted the genomic databases Human Genome Segmental Database (http://projects.tcag.ca/humandup/), DECIPHER (http:// decipher.sanger.ac.uk/), the European Cytogeneticists Association Register of Unbalanced Chromosome Aberrations (http://umcecaruca01. extern.umcn.nl:8080/ecaruca/ecaruca.jsp) and the UCSC Genome 
a

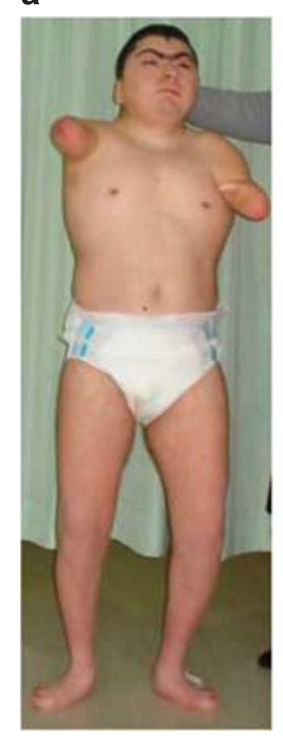

b

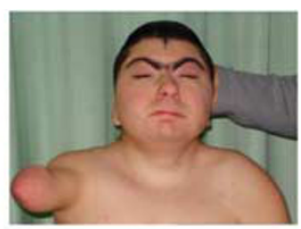

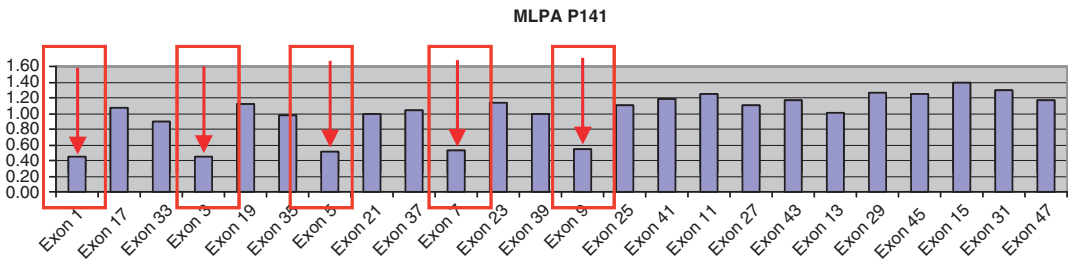

MLPA P142

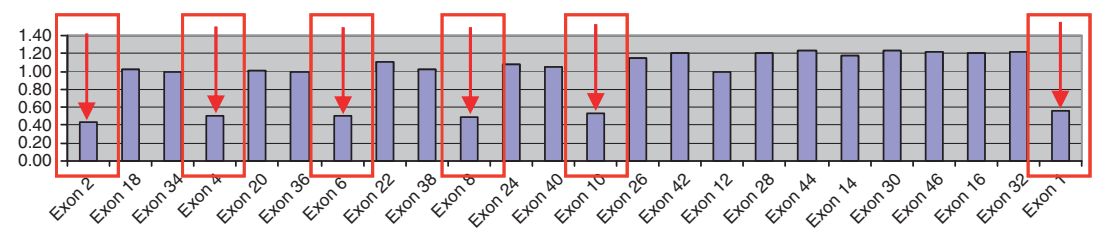

C

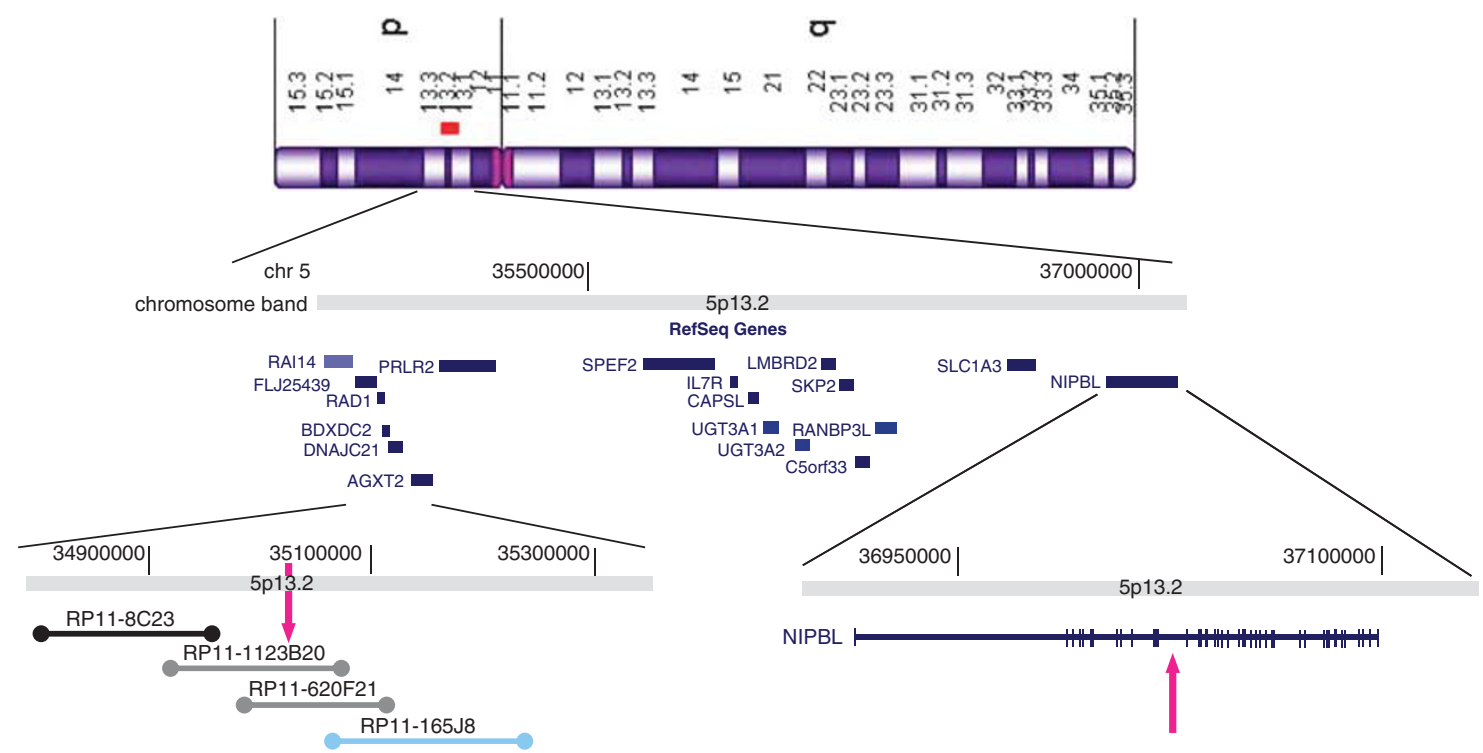

Figure 2 The clinical and genomic data of patient 1, suggesting a contiguous gene syndrome involving NIPBL. (a) Severe limb reduction and typical CdLS facial dysmorphism. (b) In the MLPA histograms, the red rectangles and arrows indicate the halved columns of the deleted exons: exon 1-10. (c) Ideogram of chromosome 5 and genomic details of the deleted $2 \mathrm{Mb}$ region at $5 \mathrm{p} 13.2$ (red bar): the BAC clones used for the FISH characterisation are represented by horizontal bars and the NIPBL gene is schematically shown with all of its exons. MLPA indicated that the first 10 exons of NIPBL are deleted. The metaphase panels showed that RP11-165J8 gave only one signal, RP11-1123B20 and RP11-620F21 gave one normal and one faint signal, and RP11$8 \mathrm{C} 23$ gave two signals mapping outside the deleted region.

Browser (http://www.genome.ucsc.edu/), none of which includes any of the reported NIBPL rearrangements.

\section{Clinical and molecular characterisation}

All the clinical details of the seven patients are summarised in Table 1.

\section{Patient 1}

Patient 1 is a 36-year-old man with typical CdLS facial features (Figure 2a), pre- and post-natal growth delay, upper limb reduction, severe intellectual disability associated with the absence of speech, deafness, and gastro-esophageal reflux (GER). His clinical score of 38 is the most severe in our CdLS cohort. To investigate whether the
MLPA-assessed deletion of exons 1-10 (Figure 2b) extends upstream of the NIPBL gene, we mapped parent-to-proband segregation of the SNPs in the $5^{\prime} \mathrm{UTR}$ region (data not shown). Of the informative markers, rs10035888 (at 36384705) mapped within the deletion, whereas rs10036454 mapped outside it, as the proband was heterozygous. The telomeric breakpoint of the deletion was refined by means of FISH analysis. As shown in Figure 2c, the region targeted by probe RP11-165J8 is deleted, that targeted by BAC RP11-8C23 is not deleted, and RP11-1123B20 and RP11-620F21 give signals of decreased intensity on one chromosome $5 \mathrm{p}$. Accordingly, the telomeric breakpoint of the deletion lies in the $30 \mathrm{~kb}$ region shared by clones RP11-1123B20 and RP11-620F21. The deletion was estimated to span $2 \mathrm{Mb}$, 


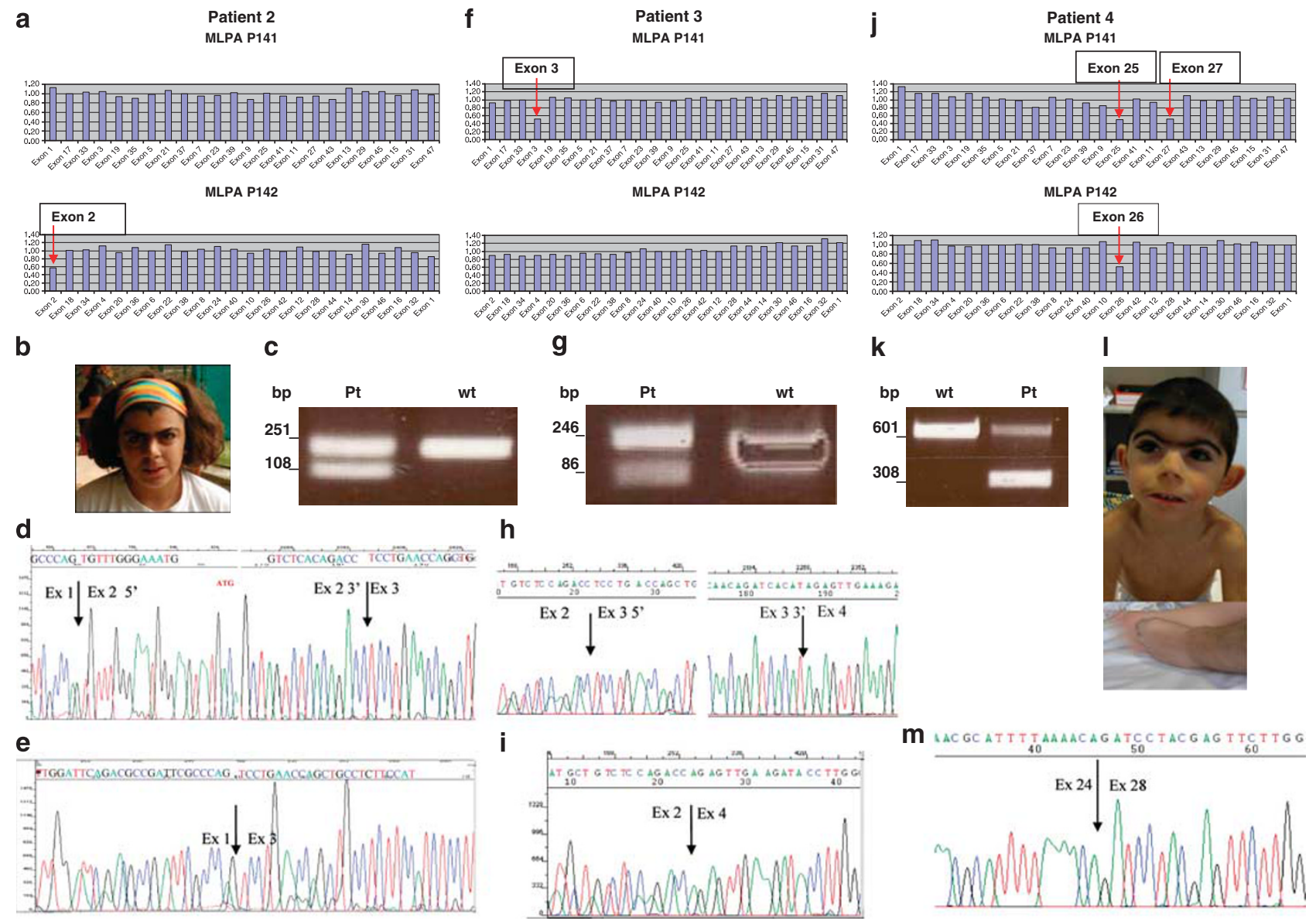

Figure $\mathbf{3}$ Vertical panels showing (from top to bottom) MLPA analysis (a, $\mathbf{f}$ and $\mathbf{j}$ ), RT-PCR (c, $\mathbf{g}$ and $\mathbf{k}$ ), and the sequencing of the wild type (d and $\mathbf{h}$ ) and aberrant (e, $\mathbf{i}$ and $\mathbf{m}$ ) transcripts of patients 2,3 , and 4 . In the MLPA histograms, the red arrows indicate the halved columns of the deleted exons: exon 2 in patient 2, exon 3 in patient 3 , and exons 25, 26, and 27 in patient 4 . All of the RT-PCRs revealed a wild type and a lower band corresponding to the deleted fragment, whose sequence indicates the exact splice-junction. The pictures of patients 2 (b) and 4 (I) show the typical facial dysmorphism of CdL syndrome.

extending for about $1.9 \mathrm{Mb}$ outside NIPBL to include the AGXT2 (alanine-glyoxilate aminotransferase 2) gene at the telomeric end and other 12 genes, one of which is SLC1A3 (Solute Carrier Family 1) that codes for the transporter molecules regulating neurotransmitter concentrations at the excitatory glutamatergic synapses of the mammalian central nervous system, and may contribute to the patient's severe behavioural disorder.

\section{Patients 2 and 3}

Patient 2 is a 16-year-old girl with facial dysmorphism (Figure $3 b$ ) obesity, mild GER, and moderate intellectual disability. Her clinical score was 20 , in the mild range, as attested by the fact that she does not show any growth delay or limb reduction, and is also able to speak. MLPA revealed that the area of exon 2 is halved (arrowed in Figure 3a), and RT-PCR detected both wild-type and aberrant transcripts (Figure 3c). Sequencing showed that the size of the aberrant band was $108 \mathrm{bp}$, thus indicating the exact skipping of exon 2 (Figures $3 \mathrm{~d}$ and e). The mutation (c.-75_+65del) leads to the removal of the ATG start codon. Patient 3 is a 3 -year-old girl with mild facial dysmorphism, post-natal growth delay, mild to moderate psychomotor delay, absent speech and GER. MLPA detected a halfdose of exon 3 (arrowed in Figure $3 \mathrm{f}$ ) corresponding to an aberrant transcript (Figure 3g) that exactly skips exon 3 (Figures $3 \mathrm{~h}$ and i). The mutation (c.65_230del) predicts a truncated pLeu22GlnfsX3 protein (Figure 1b).

\section{Patient 4}

A four-year-old boy with characteristic facial dysmorphism, pre- and post-natal growth delay, limb malformations (Figure 31), severe intellectual disability and the absence of speech. His clinical score was 23. MLPA revealed the deletion of NIPBL exons 25, 26 and 27 (Figure 3j). Direct sequencing showed that the aberrant transcript detected by RT-PCR (Figure 3k) was due to the in-frame skipping of exons 25-27. The mutation (c.4921_5328del; Figure 3m) predicts a protein lacking 135 amino acids, p.Val1641_Gln1776del (Figure 1b).

\section{Patients 5 and 6}

The deletion of patient 5 and the duplication of patient 6 both affect NIPBL exon 32. Patient 5 is a 12-year-old boy with a clinical score of 34 (severe), classic CdLS dysmorphism (Supplementary Figure 1b), severe growth delay (also recorded prenatally), kidney and genital malformations, myopia, deafness and profound intellectual disability. MLPA revealed a reduction in the area of exon 32 (arrowed in Supplementary Figure 1a), but the mutation could not be evaluated 


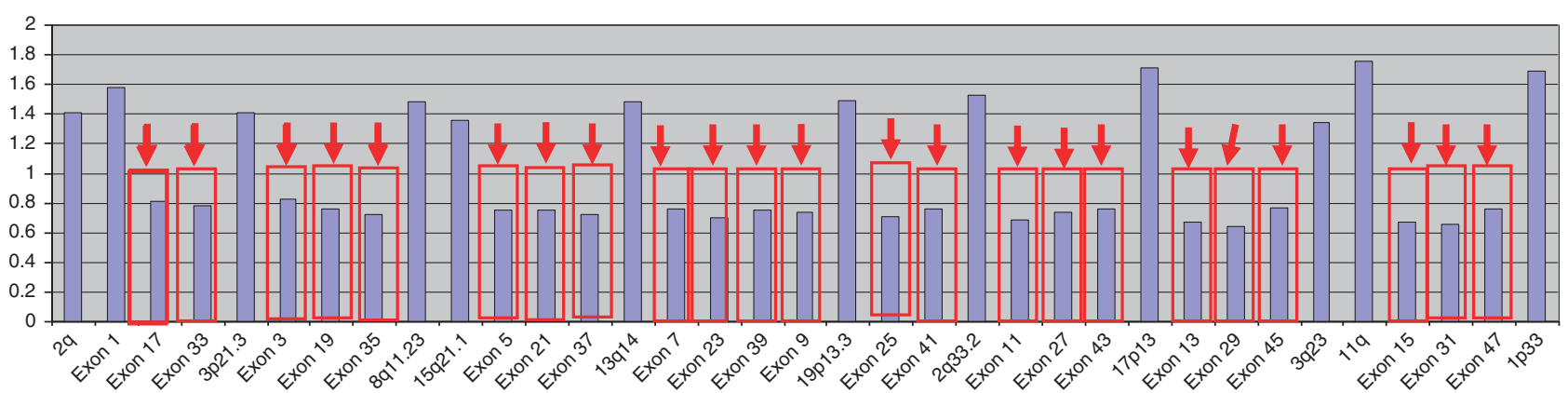

MLPA P142
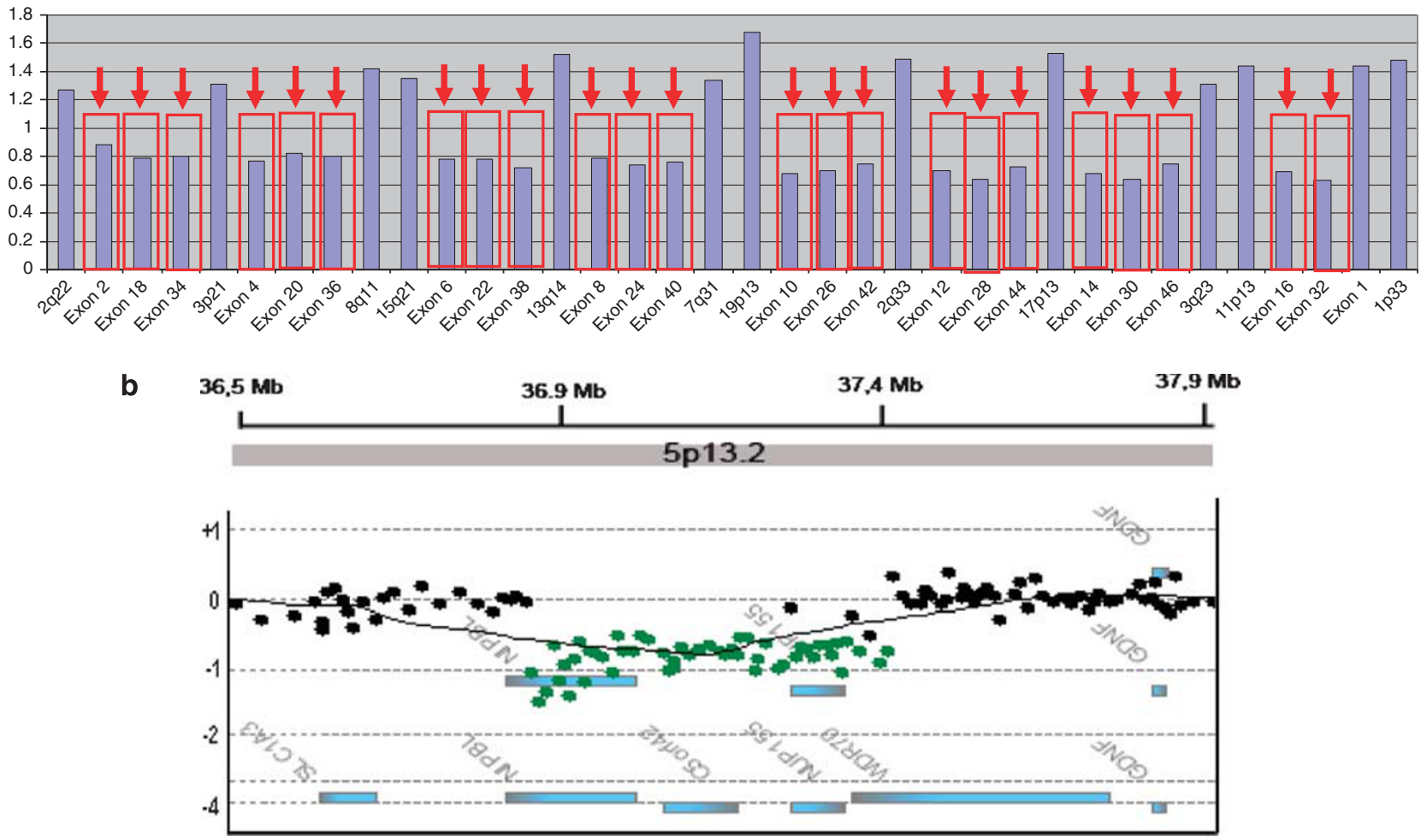

Figure 4 Clinical and genomic data of patient 7. (a) MLPA histograms: NIPBL exons arrowed in red (2-47) show reduced height in comparison with the control probes $(0.7 \mathrm{vs} 1.4)$. (b) a-CGH profile focussing on a $1.4 \mathrm{Mb}$ window within the $5 \mathrm{p} 13.2$ interval containing the NIPBL deletion; the scatter plot shows a deletion in 5 p13.2 (the shift is marked by green dots under 0 ). Each dot represents a single probe.

at transcript level. In patient 6, MLPA revealed a double dose of exon 32 (Supplementary Figure 1c). This patient was sent to our centre from South America, which is why we could not collect any clinical information suitable for assigning an overall score. Genomic q-Real time PCR confirmed the presence of a double amount of PCR product corresponding to exon 32 duplication (Supplementary Figure 1d). Amplification of this exon using exon 32 flanking primers did not reveal any longer PCR product, thus making it likely that the duplication breakpoints map within introns 31 and 33, and involves part of their sequence.

\section{Patient 7}

Patient 7 is a girl who has been suspected of having CdLS since she was 3 months old; a detailed clinical evaluation at the age of 6 years indicated a global score of 26 . She has typical facial dysmorphism, fullbody hirsutism, clinodactyly of the left little finger and limited elbow movement, absent the ring and little fingers of her right hand, gastroesophageal reflux, mild hypotrophy of the corpus callosum, and myopia with moderate growth retardation, psychomotor delay and intellectual disability. MLPA revealed control probes with values of about 1.4 and almost all of the NIPBL exons with values of about 0.7 , thus suggesting a deletion from exon 2 to the last exon of the gene (Figure 4a). The MLPA data were validated by means of a-CGH, which revealed a large deletion $(515 \mathrm{~kb}$, from kb 36950899 to 37466333 ) involving the whole NIPBL gene and extending to within the $3^{\prime}$ UTR region (chromosome 5p13.3) (Figure 4c). The telomeric deletion breakpoint maps within an $8.7 \mathrm{~kb}$ region between $36942193 \mathrm{bp}$ and $36950899 \mathrm{bp}$, whereas the centromeric breakpoint is located within an $8.9 \mathrm{~kb}$ interval between $37466333 \mathrm{bp}$ and $37475302 \mathrm{bp}$. The deleted region includes most of the coding sequence of NIPBL (from exon 2 to the 3'UTR) plus C5orf 42 (chromosome 5 open reading frame 42 for a putative transmembrane protein), NUP155 (coding for a nucleoporin protein) and part of the WDR70 gene containing a domain homologues to WDR40. The clinical 
presentations of the CdLS patients whose NIPBL rearrangements were revealed by MLPA are very heterogenous, with clinical scores ranging from the severe scores of patients 1 and 5 (38 and 32) to the moderate scores of patients 4 and 7 (23 and 26) and the very mild scores of patients 2 and 3 (20 and 18). Only the carriers of large deletions (1 and 7) show limb reduction or anomalies, whereas all of the patients except patient 3 who has a normal growth curve are characterised by mild-severe intellectual disability, typical facies and growth retardation. Patient 6 is not included in this comparative overview because of the lack of clinical details.

\section{DISCUSSION}

A comprehensive NIPBL locus-specific mutation database has recently been set up using LOVD (Oliveira et al ${ }^{25} \mathrm{http}: / /$ www.lovd.nl/NIPBL), which among the different mutation types identified in 196 CdLS patients, assigns decreasing frequencies to small deletions $(27.6 \%)$, missense $(21.1 \%)$, nonsense (17.3\%) and splicing mutations (17.1\%), and small duplications (13.6\%). There are few insertions and indels (1.5\% each), and gross deletions/duplications are limited to the two described by Bhuiyan et $a l^{12}$ and Vrouwe et al. ${ }^{11}$ Our cohort of 200 investigated CdLS patients shows a mutation distribution that is quite similar to that of the LOVD database, except for the higher frequency of splicing defects $(33.8 \%)$. Whenever we could investigate the effects of splice mutations, we have always found that the causative lesion was the lack of a whole exon (data not shown). This was the rationale for the present study as it favoured the view that whole-exon deletions with effects similar to splicing defects might be responsible for CdLS in a subset of patients. The use of MLPA to test samples from a group of 132 CdLS patients who were negative to the standard NIPBL test validated this hypothesis by revealing four intragenic single- or multi-exon deletions and one single-exon duplication. MLPA also identified two unusually large NIPBL deletions.

Interestingly, the extent and gene content of the deletion carried by patient 1 allows to classify him as a first reported carrier of a contiguous gene syndrome, including NIPBL. Two instances of deletions of chromosome 5 p13 involving NIPBL have been reported in CdLS patients, ${ }^{17,18}$ both of which were cytogenetically detectable and occurred in infants who died perinatally, thus preventing a full description of their clinical presentation and the precise mapping of the deletion breakpoints. Three large NIPBL imbalances (all detected by means of MLPA) have subsequently been reported, ${ }^{11-13}$ two of which are cited in the LOVD NIPBL-specific database, but all appear to be intragenic. On the basis of these data and those of the present study, it seems that, although rare, large rearrangements affecting NIPBL may occur, as was predicted by the results of a study based on a 'reverse genomics approach' that did not focus on CdLS patients. ${ }^{16}$ This study, which had the aim of establishing the dose effect of cohesin-associated genes by means of targeted oligonucleotide a-CGH analyses, found five patients carrying duplications on the chromosome 5 region containing NIPBL, four of which extended beyond the NIPBL gene. ${ }^{16}$ Our study shows that large NIPBL deletions can also be detected by means of MLPA. The large multigenic deletion of patient 1 could be correlated to his extremely severe phenotype. This de novo deletion spans about $2 \mathrm{Mb}$ from the centromeric breakpoint within NIPBL to the telomeric breakpoint (13 genes), as indicated by the complementary use of MLPA, FISH and SNP/microsatellite analyses. Physical examination showed the presence of all of the signs of CdLS, including pre- and post-natal growth retardation, typical facial dysmorphism (Figure 2a), delayed psychomotor development, severe intellectual disability, bilateral severe upper limb reduction (Figure 2a), small feet, deafness and gastroesophageal reflux. This severe presentation is probably due to the combined haploinsufficiency of dose-sensitive genes located in the deleted region (Figure $2 \mathrm{~b}$ ). One relevant gene may be $S L C 1 A 3$, which encodes a member of a family of high-affinity sodium-dependent transporter molecules that regulate neurotransmitter concentrations at the excitatory glutamatergic synapses of the mammalian CNS. ${ }^{19}$ Glutamate and aspartate are excitatory neurotransmitters involved in various CNS diseases. Heterozygous mutations in the SLC1A3 gene have been reported in one sporadic case and in three members of a family affected by type 6 episodic ataxia, ${ }^{20,21}$ and SLCA3 was included in the duplication of one of the five patients with intellectual disability, developmental delay and severe behavioural abnormalities described by Yan et al. ${ }^{16}$ We hypothesise that SLC1A3 may contribute to the neurological impairment of our patient 1 (which is extreme even among CdLS patients), and minimise the role of the other genes. Interpreting the effect of the loss of a single gene mapping to a deleted region is very difficult, especially when the deletion is too large to identify the specific contribution of each gene to the phenotype. Nevertheless, as the patient has all of the signs of CdLS without any definitive extraCdLS signs, it seems that NIPBL is the major dose-sensitive gene. Despite its more limited extension, the deletion of patient 7 is similar insofar as it involves the whole of NIPBL gene and 3'UTR regions that contain ORFs whose functional roles are still unclear, the NUP155 gene, and part of the interrupted WDR70 gene encoding for a WD repeat containing protein 70 , which is found in a number of eukaryotic proteins with a wide variety of functions, including adaptor/regulatory modules in signal transduction, pre-mRNA processing and cytoskeleton assembly, and typically contains a GH dipeptide 11-24 residues. The proband has a classic CdLS phenotype with a moderate/severe score and partial limb reduction, all of which are signs attributable to the absence of NIPBL. Interestingly, three of the five patients described by Yan et $a l^{16}$ and carrying NIPBL duplications also included these genes, and one of them had short fifth fingers. The centromeric breakpoint of our patient 7 falls between bp 37466333 and bp 37475302 , very close to the centromeric breakpoint of the reported duplications, which map between 37083320 and 37420236 . We hypothesise that both reciprocal rearrangements may affect the function of the NUP155 gene, although the contribution to the clinical phenotype of our patient is difficult to define. Interestingly, the closeness of the centromeric breakpoint of our patient to those reported by Yan et $a l^{16}$ suggests that these rearrangements may be mediated by common genomic mechanisms primed by DNA sequence motifs.

As a whole, our findings indicate that MLPA can make a substantial contribution to defining the entire mutational repertoire of NIPBL featuring as unique approach in detecting NIPBL intragenic single or multi-exon deletions and duplications that might be overlooked by a-CGH, as exemplified by patients 2, 3, 5 and 6 who would have never been disclosed by a-CGH. The intragenic exon 3 deletion of patient 3 (which cDNA analysis indicates as predicting protein truncation) deserves comment because this exon encodes a delangin domain that interacts with the Mau-2 protein required for the binding of cohesin to chromosomes. ${ }^{22}$ The consequence of the deletion (p.Leu22GlnfsX3) is the same as that predicted by the nonsense mutation described in the literature ${ }^{23,24}$ and the splicing mutation (c.65-5A.G) found in two of our CdLS patients ${ }^{7}$ (plus unpublished data). Patients 2 and 3, whose deletions skip exons within the NIPBL amino terminus, have a very mild phenotype (clinical scores of 20 and 18) even though it is predicted that they are haploinsufficient for the protein. Whereas patient 4 , who has an in-frame deletion damaging the HEAT domain, has a severe phenotype. It may be that the absence of a truncated protein is less damaging than the expression of an 
aberrantly truncated protein or that some other factors interact to modulate the complex CdLS phenotype. Unlike the Oliveira database, in which exon 32 does not seem to harbour any mutations in CdLS probands, ${ }^{25}$ MLPA revealed two rearrangements involving exon 32 in our cohort (one deletion and one duplication), thus showing its usefulness in delineating the NIPBL mutational spectrum. Although exon 32-encoded protein includes 54 amino acids, we could not establish the in-frame occurrence of the deletion by means of transcript analysis, but the severe phenotype of patient 5 can be explained by the inclusion of the HEAT domain in the aberrant protein. The use of MLPA analysis after the standard NIPBL test identified a further $5.3 \%$ of genetic lesions accounting for CdLS in $N I P B L$-negative patients, including seven deletions (four intragenic and two extending beyond the NIPBL gene), and one duplication. When applied to CdLS patients per se, MLPA is capable of detecting the molecular lesion in 3.5\% (7/200) of the patients. For these reasons, we strongly encourage the inclusion of MLPA as an obligatory step in NIPBL test. We have applied MLPA to a cohort of CdLS patients, negative to both $N I P B L$ and $S M C 1 A$ genes, which has been cumulated across time. However, according to the gained experience, we believe that the diagnostic flow chart of patients with a consistent clinical diagnosis of CdLS should start with the standard test of the major NIPBL gene, be then completed by NIPBL MLPA test and continued by the screening of SMC1A gene. The a-CGH approach overlaps MLPA in detecting NIPBL large rearrangements, but is less sensitive than MLPA in detecting small intragenic losses/gains. Conversely, a-CGH can be pursued especially in patients with a CdLS-like clinical presentation, being suitable to detect imbalances of genomic regions other than those harbouring the currently known $C d L S$ genes.

\section{CONFLICT OF INTEREST}

The authors declare no conflict of interest.

\section{ACKNOWLEDGEMENTS}

We would like to thank the patients' families for participating in this study, and acknowledge the support of ISS rare disease Grant 526/A27 to LL, project 'Clinical, functional, behavioural and genetic characterisation of new candidate genes of the cohesin-condensin complex'.

1 Krantz ID, McCallum J, DeScipio C et al: Cornelia de Lange syndrome is caused by mutations in NIPBL, the human homolog of Drosophila melanogaster Nipped-B. Nat Genet 2004; 6: 631-635.

2 Tonkin ET, Wang TJ, Lisgo S, Bamshad MJ, Strachan T: NIPBL, encoding a homolog of fungal Scc2-type sister chromatid cohesion proteins and fly Nipped-B, is mutated in Cornelia de Lange syndrome. Nat Genet 2004; 36: 636-641.

3 Musio A, Selicorni A, Focarelli ML et al: X-linked Cornelia de Lange syndrome owing to SMC1L1 mutations. Nat Genet 2006; 38: 528-530.
4 Yan J, Saifi GM, Wierzba TH et al: Mutational and genotype-phenotype correlation analysis in 28 polish patients with Cornelia de Lange syndrome. Am J Med Gen 2006; 140: 1531-1541.

5 Bhuiyan ZA, Klein M, Hammond P et al: Genotype-phenotype correlations of 39 patients with Cornelia de Lange syndrome: the Dutch experience. J Med Gen 2006; 43: 568-575.

6 Deardorff MA, Kaur M, Yaeger D et al: Mutations in cohesin complex members SMC3 and SMC1A cause a mild variant of Cornelia de Lange syndrome with predominant mental retardation. Am J Hum Genet 2007; 80: 485-494.

7 Selicorni A, Russo S, Gervasini C et al: Clinical score of 62 Italian patients with Cornelia de Lange syndrome and correlations with the presence and type of NIPBL mutation. Clin Genet 2007; 72: 98-108.

8 Hayashi S, Ono M, Makita Y, Imoto I, Mizutani S, Inazawa J: Fortuitous detection of a submicroscopic deletion at 1q25 in a girl with Cornelia de Lange syndrome carrying $\mathrm{t}(5 ; 13)(\mathrm{p} 13.1 ; \mathrm{q} 12.1)$ by array-based comparative genomic hybridization. Am J Med Genet A 2007; 143A: 1191-1197.

9 Schoumans J, Wincent J, Barbaro $\mathrm{M}$ et al: Comprehensive mutational analysis of a cohort of Swedish Cornelia de Lange syndrome patients. Eur J Hum Genet 2007; 15: 143-149.

10 Gervasini C, Pfundt R, Castronovo P et al: Search for genomic imbalances in a cohort of 24 Cornelia de Lange patients negative for mutations in the NIPBL and SMC1L1 genes. Clin Genet 2008; 74: 531-538.

11 Vrouwe MG, Elghalbzouri-Maghrani E, Meijers M et al: Increased DNA damage sensitivity of Cornelia de Lange syndrome cells: evidence for impaired recombinational repair. Hum Mol Genet 2007; 16: 1478-1487.

12 Bhuiyan ZA, Stewart H, Redeker EJ, Mannens MM, Hennekam RC: Large genomic rearrangements in NIPBL are infrequent in Cornelia de Lange syndrome. Eur J Hum Genet 2007; 15: 505-508.

13 Ratajska M, Wierzba J, Pehlivan D et al: Cornelia de Lange syndrome case due to genomic rearrangements including NIPBL. Eur J Med Genet 2010; 53: 378-382.

14 Kline AD, Krantz ID, Sommer A et al: Cornelia de Lange syndrome: clinical review, diagnostic and scoring systems, and anticipatory guidance. Am J Med Genet A 2007; 143A: 1287-1296.

15 Lichter $\mathrm{P}$, Cremer T: Chromosome analysis by non-isotopic in situ hybridization; in: Rooney DE and Czipolkowski BH (eds):: Human Cytogen-A Practical Approach. Oxford: University Press, 1992, pp 157-192.

16 Yan J, Zhang F, Brundage E et al: Genomic duplication resulting in increased copy number of genes encoding the sister chromatid cohesion complex conveys clinical consequences distinct from Cornelia de Lange. J Med Genet 2009; 46: 626-634

17 Taylor MJ, Josifek K: Multiple congenital anomalies, thymic dysplasia, severe congenital heart disease, and oligosyndactyly with a deletion of the short arm of chromosome 5. Am J Med Genet 1981; 9: 5-11.

18 Hulinsky R, Byrne JL, Lowichik A, Viskochil DH: Fetus with interstitial del(5)(p13.1p14.2) diagnosed postnatally with Cornelia de Lange syndrome. Am J Med Genet A 2005; 137A: 336-338.

19 Kirschner MA, Arriza JL, Copeland NG et al: The mouse and human excitatory amino acid transporter gene (EAAT1) maps to mouse chromosome 15 and a region of syntenic homology on human chromosome 5 . Genomics 1994; 22: 631-633.

20 Jen JC, Wan J, Palos TP, Howard BD, Baloh RW et al: Mutation in the glutamate transporter EAAT1 causes episodic ataxia, hemiplegia, and seizures. Neurology 2005; 65: 529-534.

21 de Vries B, Mamsa $\mathrm{H}$, Stam AH et al: Episodic ataxia associated with EAAT1 mutation C186S affecting glutamate reuptake. Arch Neurol 2009; 66: 97-101.

22 Dorsett D, Krantz ID: On the molecular etiology of Cornelia de Lange syndrome. Ann NY Acad Sci 2009; 1151: 22-37.

23 Gillis LA, McCallum J, Kaur M et al: NIPBL mutational analysis in 120 individuals with Cornelia de Lange syndrome and evaluation of genotype-phenotype correlations. Am J Hum Gene 2004; 75: 610-623.

24 Pié J, Gil-Rodríguez MC, Ciero $\mathrm{M}$ et al: Mutations and variants in the cohesion factor genes NIPBL, SMC1A, and SMC3 in a cohort of 30 unrelated patients with Cornelia de Lange syndrome. Am J Med Genet A 2010; 152A: 924-929.

25 Oliveira J, Dias C, Redeker E et al: Development of NIPBL locus-specific database using LOVD: from novel mutations to further genotype-phenotype correlations in Cornelia de Lange syndrome. Hum Mutat 2010; 31: 1216-1222.

Supplementary Information accompanies the paper on European Journal of Human Genetics website (http://www.nature.com/ejhg) 